\title{
A rare case of discontinuous type splenogonadal fusion in a 13 year old boy: a case report and review of literature
}

\author{
Asmir Jonuzi ${ }^{1 *}$, Nusret Popović1, Zlatan Zvizdić1, Emir Milišić1, Melika Bukvić², \\ Irmina Sefićc ${ }^{2}$, Amra Džananović ${ }^{2}$, Amira Mesic ${ }^{3}$, Adisa Chikha ${ }^{4}$, Amila Zukanović ${ }^{5}$, \\ Benjamin Kulovac ${ }^{6}$
}

\author{
${ }^{1}$ Department of Pediatric surgery, Clinical Center University in Sarajevo, Patriotske lige Sarajevo, Bosnia and \\ Herzegovina \\ ${ }^{2}$ Department of Radiology, Clinical Center University in Sarajevo, Bolnička, Sarajevo, Bosnia and Herzegovina \\ ${ }^{3}$ Department of Anaesthesiology and Reanimation, Clinical Center University in Sarajevo, Bolnička, Sarajevo, Bosnia \\ and Herzegovina \\ ${ }^{4}$ Department of Pathology, Clinical Center University in Sarajevo, Bolnička, Sarajevo, Bosnia and Herzegovina \\ ${ }^{5}$ Faculty of Dentistry with Clinics University of Sarajevo, Bolnička, Sarajevo, Bosnia and Herzegovina \\ ${ }^{6}$ Department of Urology, Clinical Center University in Sarajevo, Bolnička, Sarajevo, Bosnia and Herzegovina
}

Received: 27 July 2020

Accepted: 05 September 2020

\section{*Correspondence:}

Dr. Asmir Jonuzi,

E-mail: jonuziasmir@hotmail.com

Copyright: () the author(s), publisher and licensee Medip Academy. This is an open-access article distributed under the terms of the Creative Commons Attribution Non-Commercial License, which permits unrestricted non-commercial use, distribution, and reproduction in any medium, provided the original work is properly cited.

\section{ABSTRACT}

Splenogonadal fusion (SGF) is a rare developmental anomaly in which an abnormal connection between the splenic tissue and gonads or mesonephric derivatives is present. This entity often presents with scrotal mass, inguinal hernia, or cryptorchidism. Less than 200 cases have been reported since it was first described in 1883 . It can be of continuous and discontinuous type based on the presence of a band of connecting splenic tissue. Report a rare case of discontinuous type of SGF in an adolescent male presenting as nonpalpable testis. On evaluation, ultrasonography (USG) and magnetic resonance imaging of abdomen and pelvis, his left scrotal testis was atrophied and right intra-abdominal undescended testis. This is the first reported case of SGF from Bosnia and Herzegovina. Laparoscopy was demonstrated to be the only accurate exploratory procedure for the diagnosis and surgical treatment of SGF with non-palpable testis.

Keywords: Cryptorchidism, Developmental anomaly, Laparascopy, Splenogonadal fusion

\section{INTRODUCTION}

SGF is a rare congenital anomaly characterized by fusion of the spleen and a gonad. Less than 200 cases have been reported since it was first described in 1883 by Bostroem. ${ }^{1}$ There are two types of SGF-continuous and discontinuous. The continuous type involves a direct anatomic connection between the spleen and gonad by means of a cord made of splenic and/or fibrous tissue. The cord may be attached to any area of the splenic hilum or to the inferior pole of the spleen. The discontinuous type is characterized simply by gonadal fusion with an accessory spleen or ectopic splenic tissue. ${ }^{2}$

Such rare anomaly usually takes place during the 5th to 8th week of gestation, that is, before gonadal descent starts and presents as mass. The product fusion commonly diagnosed as a testicular mass of unknown origin in association with other congenital deformities. ${ }^{1}$

Only a few cases have been diagnosed preoperatively, which were typically regarded as cryptorchidism, 
testicular tumors or inguinal hernia, and accidentally determined to be SGF after exposure of the inguinal canal during surgery. ${ }^{3}$ The condition affects young males. More than $70 \%$ of affected patients are younger than 20 years, and approximately $50 \%$ are paediatric patients. ${ }^{4}$

Report a case of discontinuous-type, left-sided SGF in a 13-year-old male, discovered during laparoscopic exploration nonpalpable testis.

\section{CASE REPORT}

General physical examination was normal and there were no congenital anomalies. On local examination, patient's left atrophic testis was situated in scrotum and of size 0.5 $\mathrm{cm} \times 0.5 \mathrm{~cm}$. On the other hand, there was empty scrotum on right side which was suggestive of undescended testes. There were no associated congenital anomalies seen. He had no notable past medical history, and there was no history of genitourinary trauma or urinary symptoms. $\mathrm{He}$ was the product of a normal pregnancy and well developed for his age. Routine blood investigations, renal function test, and liver function tests were normal. The levels of tumor markers as AFP and beta-hCG were normal. USG revealed absence of right sided testis and epididymis in the scrotum and inguinal canal. Magnetic resonance imaging (MRI) reported five oblong masses each $1.5 \times 1 \mathrm{~cm}$ in the left retroperitoneal region anterior and lateral to psoas muscle (Figure 1). Patient was taken for laparoscopic assessment and was found to have right sided testicular mass with no lymph nodal enlargement. On careful examination, it was suggestive for SGF anomaly for which he underwent left orchidectomy and five oblong masses (Figure 2).

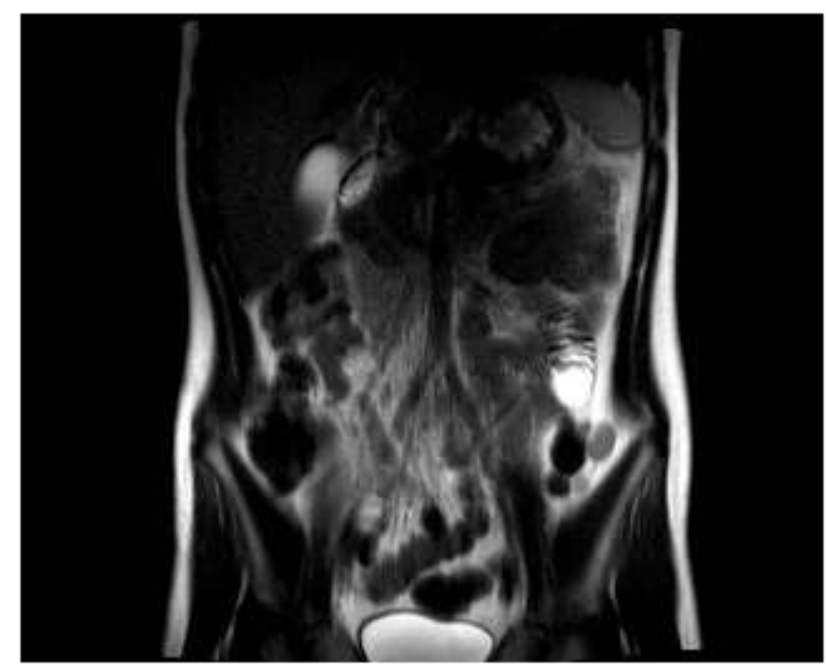

Figure 1: MRI T2, sagittal cuts of 5 oblong masses in the left retroperitoneum.

Distally, the cord-like splenic tissue was attached to the testis, and proximally, it was discontinuous and connected to the principal spleen. The specimen (Figure 3) was sent for histopathology which showed the tumor mass to be composed of fibrous capsule with red and white pulp within it suggestive of spleen and there was no evidence of spermatogenesis in the testicular portion of resected specimen (Figure 4). He fared well in postoperative period and was discharged on postoperative day 5. At the 2-years follow-up, the patient had no problems and the right testis was observed to have retained its normal structure.

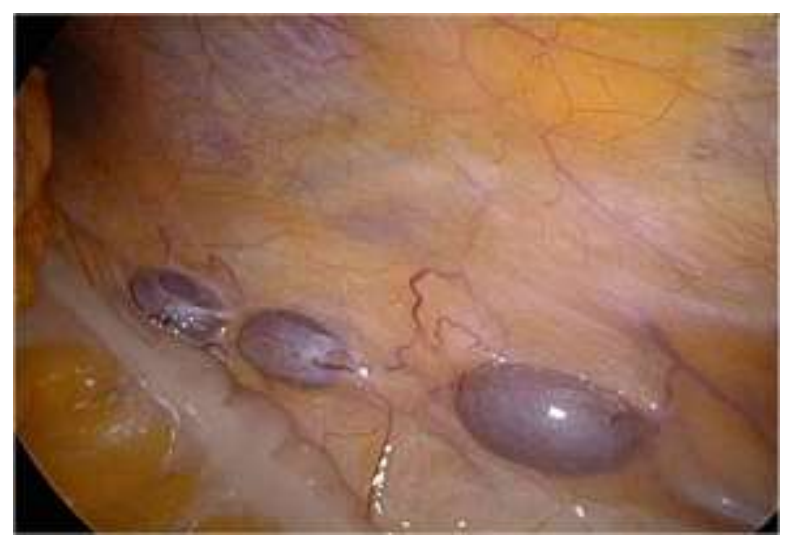

Figure 2: Laparoscopic assessment of SGF.

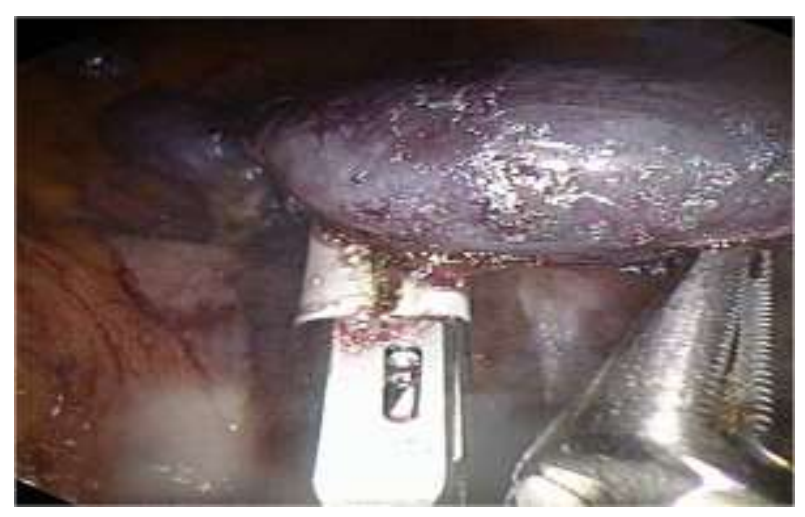

Figure 3: Gross appearance of the specimen.

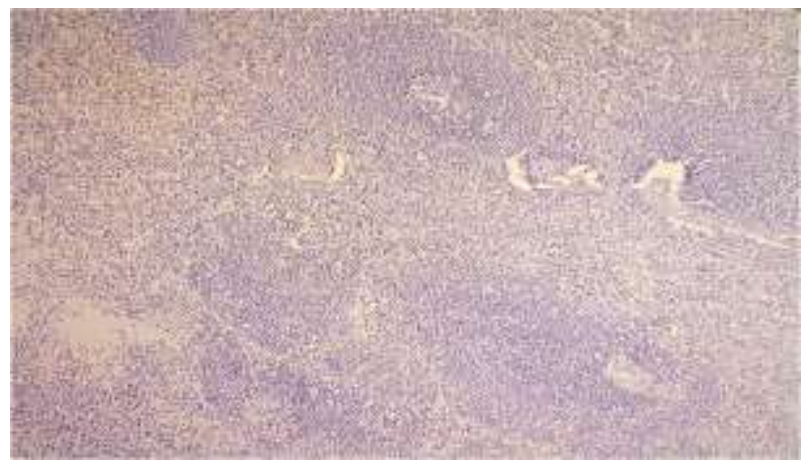

Figure 4: Microscopic picture of spleen of white and red pulp in mass ( $\mathrm{H}$ and $\mathbf{E}$ stain $\times \mathbf{1 0 0}$ magnification).

\section{DISCUSSION}

SGF is a rare congenital anomaly, between the spleen and a gonad or mesonephric derivatives. The first description of SGF was made by Bostroem in 1883, and a detailed 
report by Pommer followed in $1889 .{ }^{1}$ Less than 200 cases have been reported worldwide since its first description. In 1990, a detailed literature review of 123 cases was conducted by Carragher. ${ }^{5,6}$ This entity is almost entirely left sided $(98 \%)$ and demonstrates a profound male predominance (incidence 16:1). ${ }^{7}$ In 1956 Putschar and Manion classified SGF into two groups: continuous and discontinuous. In the continuous type, a cord of splenic tissue, a fibrous cord beaded with splenic nodules or a pure fibrous band anatomically connects the main spleen to the gonad. This cord may traverse the peritoneal cavity to enter the inguinal canal or remain in the paracolic gutter. In the discontinuous type, the ectopic splenic tissue would be adherent to the gonads with no anatomical connections to the principal spleen. Carragher reported that both types occur with equal frequency. ${ }^{1}$ Continuous SGF is commonly found in association with a range of other malformations, such as cleft palate, micrognathia, cardiac defects, limb deformities, and anal malformations. ${ }^{8,9}$ The splenic tissue is often found within the tunica vaginalis and is closely associated with the testicular parenchyma. However, a fibrous capsule often is present and prevents free intermingling of tissues. ${ }^{8}$ The exact pathogenesis for SGF is not yet understood, but it is thought to develop between the fifth and the eighth week of gestation based on the close location between the splenic tissue and left gonadal ridge. When the spleen develops in the dorsum of the mesogastrium forma group of mesenchymal cells, the gonads start to develop in the posterolateral wall of the embryo at the same time. During embryonic gut rotation, the spleen development comes into close approximation to the left urogenital fold. Furthermore, subsequent inflammation or adhesions on the peritoneal surface may facilitate splenogonadal fusion. ${ }^{10-12}$ SGF is seen primarily in young males, who present with a scrotal mass, inguinal hernia, or undescended testis. Cryptorchidism is the most common anomaly associated with SGF, and $59 \%$ of patients have bilateral undescended testes. ${ }^{13}$ Rarely, traumatic rupture of the testis may occur, sometimes with infectious diseases such as mumps, malaria, and mononucleosis, or intestinal obstruction may be caused by the splenic tissue attached to the spermatic cord. ${ }^{14}$ Atrophy and hypoplasia may be seen in the testis tissue adjacent to the lesion. There have been a few reports of discontinuous SGF associated with ipsilateral testicular atrophy. ${ }^{15-17}$ Most cases of SGF have been reported with ipsilateral and rarely with contralateral cryptorchidism. ${ }^{16,17}$ About ten cases were associated with bilateral cryptorchidism. ${ }^{18,19}$ Various imaging modalities can be very helpful but often are not performed due to low preoperative suspicion. Doppler USG of the test is often demonstrating a homogeneous echogenic vascular mass situated on the superior aspect of the testes. In terms of management, if clinical diagnosis of SGF is definite, surgical exploration is not recommended in cases of small asymptomatic mass and such patients should be followed by regular physical examination, tumor markers, and ultrasound. If surgery is performed, using intraoperative frozen section pathology if available is of utmost importance and testis can often be spared if the mass is small and separable from testis and remaining testicular tissue is worth to preserve. However, radical orchidectomy is the gold standard treatment of any solid testis mass when there is a normal contralateral testis present. It has been reported that 25$35 \%$ of SGF will necessitate orchiectomy because of testicular atrophy or inseparability of tissues. ${ }^{7,8}$ This was the case for the patient where the testicular remnant was very much atrophied and later found to be of primitive histology. This was probably due to pressure applied on the testicular parenchyma by the splenic tissue enclosed within the unyielding tunica vaginalis. However, Le Roux et al suggested that discontinuous-type SGF is a rare variant of accessory spleen. About a sixth of all cases of SGF were found during autopsies. ${ }^{2,20}$ Several cases of testicular malignancies in undescended testis associated with SGF have been reported. ${ }^{21,23}$ The lack of awareness of SGF is a major factor in its misdiagnosis. Imaging methods, including B-type USG, computed tomography (CT), MRI and 99TCm spleen scanning, aid with the diagnosis of SGF. However, laparoscopies have achieved improved diagnoses and management of SGF. ${ }^{24}$ Diagnosis of SGF is made by USG, CT, MRI and 99TCm spleen scintigraphy. Additionally, diagnosis and management of SGF were also achieved by laparoscopic procedures. ${ }^{24}$ The incidence of testicular tumor is reported as 1,6 per 100,000 for boys less than 15 years of age. $^{25}$

\section{CONCLUSION}

In conclusion, $\mathrm{SGF}$ is a rare benign urologic malformation with equivocal clinical presentations that may mislead clinicians. As basic imaging modalities may not aid in the accurate diagnosis of this condition, it is critical that both paediatric and urological surgeons are aware of this disease entity so that an early and accurate diagnosis may be reached, thus avoiding unnecessary orchidectomies; laparoscopic exploration is therefore helpful in diagnosis and treatment of patients with high cryptorchidism.

\section{Funding: No funding sources \\ Conflict of interest: None declared \\ Ethical approval: Not required}

\section{REFERENCES}

1. Carragher AM. One hundred years of splenogonadal fusion. Urology. 1990;35(6):471-5.

2. Duncan WL, Barraza MA. Splenogonadal fusion: a case report and review of literature. J Pediatr Surg. 2005;40(12):e5-7.

3. Lopes RI, de Medeiros MT, Arap MA, Cocuzza M, Srougi M, Hallak J. Splenogonadal fusion and testicular cancer: case report and review of the literature. Einstein (Sao Paulo). 2012;10(1):92-5.

4. Keyik B, Yanik B, Conkbayir I, Tuygun C, Kizilgoz V, Hekimoğlu B. Continuous-type splenogonadal fusion associated with an ipsilateral testicular 
atrophy: sonographic findings. J Clin Ultrasound. 2010;38(3):161-3.

5. Lin CS, Lazarowicz JL, Allan RW, Maclennan GT. Splenogonadal fusion. J Urol. 2010;184(1):332- 3.

6. Chiaramonte C, Siracusa F, Li Voti G. Splenogonadal Fusion: A Genetic Disorder?-Report of a Case and Review of the Literature. Urol Case Rep. 2014;2(2):67-9.

7. Huang G, Huang Y, Zeng L, Yuan M, Wu Y, Huang L. Continuous-type splenogonadal fusion: A case report. Exp Ther Med. 2017;13(5):2019-21.

8. Uglialoro AD, Goltzman ME, Niazi M, Lehman D, Silletti J, Bjurlin MA. Splenogonadal Fusion Presenting as an Asymptomatic Testicular Mass. Urology. 2016;97:1-4.

9. Alivizatos G, Skolarikos A, Sopilidis O, Ferakis N, Chorti M. Splenogonadal fusion: report of a case and review of the literature. Int J Urol. 2005;12(1):90-2.

10. Melikov R, Altunkol A, Quliyev F, Mammadov E, Abat D. Continuous- type splenogonadal fusion: a rare cause of scrotal swelling. Pediatr Urol Case Rep. 2015;2(02):22.

11. Ferrón SA, Arce JD. Discontinuous splenogonadal fusion: new sonographic findings. Pediatr Radiol. 2013;43(12):1652-55.

12. Chen SL, Kao YL, Sun HS, Lin WL. Splenogonadal fusion. J Formos Med Assoc. 2008;107(11):892-5.

13. Khairat ABM, Ismail AM. Splenogonadal fusion: case presentation and literature review. J Pediatr Surg. 2005;40(8):1357-60.

14. Putschar WG, Manion WC. Splenicgonadal fusion. Am J Pathol. 1956;32(1):15-33.

15. Nimkin K, Kleinman PK, Chappell JS. Abdominal ultrasonography of splenogonadal fusion. J Ultrasound Med. 2000;19(5):345-7.

16. Hizli F, Uygur MC, Irkkan C. Splenogonadal fusion: report of a case. Int J Urol. 2005;12(6):591-2.
17. Khelif K, Maassarani F, De Keuleneer R, Segers V. Splenogonadal fusion: a case report. Acta Chir Belg. 2010;110(6):607-8.

18. Molaeian M, Shojaei H. Splenogonadal fusion presented with cryptorchidism. Urol J. 2009;6(2):130-1.

19. Al-Marhoon M, Matthew J, Nirmala V, Kehinde EO. Splenogonadal fusion associated with primary male infertility. BJU Int. 2000;86(4):560-1.

20. Le Roux PJ, Heddle RM. Splenogonadal fusion: is the accepted classification system accurate? BJU Int. 2000;85(1):114-5.

21. Karaman MI, Gonzales ET. Splenogonadal fusion: report of 2 cases and review of the literature. J Urol. 1996 Jan;155(1):309-11.

22. Falkowski WS, Carter MF. Splenogonadal fusion associated with an anaplastic seminoma. J Urol. 1980;124(4):562-4.

23. Imperial SL, Sidhu JS. Nonseminomatous germ cell tumor arising in splenogonadal fusion. Arch Pathol Lab Med. 2002;126(10):1222-5.

24. Papparella A, Nino F, Coppola S, Donniacono D, Parmeggiani P. Laparoscopy in the diagnosis and management of splenogonadal fusion: case report. Eur J Pediatr Surg. 2011;21(3):203-4.

25. Chung JM, Lee SD. Overview of pediatric testicular tumors in Korea. Korean J Urol. 2014;55(12):78996.

Cite this article as: Jonuzi A, Popović N, Zvizdić Z, Milišić E, Bukvić M, Sefić I, et al. A rare case of discontinuous type splenogonadal fusion in a 13 year old boy: a case report and review of literature. Int Surg J 2020;7:3438-41. 